\title{
Efeitos hemodinâmicos da tiletamina-zolazepam em cães hipovolêmicos
}

\section{Hemodynamic effects of tiletamine-zolazepan in hypovolemic dogs}

\author{
Anderson Farias, ${ }^{\star}$ Carlos Eduardo Pacchini, ${ }^{\star \star}$ Carlos Augusto Araújo Valadão***
}

\begin{abstract}
Resumo
A cetamina possui ação estimulante sobre a função cardiovascular, propiciando uma estabilidade hemodinâmica em cães hipovolêmicos. Neste estudo, avaliaram-se os efeitos da associação tiletamina/zolazepam (TZ) em 14 cães divididos em grupo $A$ (normal, $n=7$ ) e grupo $B$ (hipovolêmico, $n=7$ ), os quais foram anestesiados com sevofluorano (1,5 CAM), para a implantação dos cateteres venosos, arteriais e da termodiluição, e logo após a concentração anestésica foi reduzida para (0,5 CAM). Nos cães do grupo B induziu-se hipovolemia, retirando sangue na proporção de 3\% do peso corpóreo. Aplicaram-se 10 $\mathrm{mg} / \mathrm{kg}$ de TZ, pela via intravenosa, em ambos os grupos. Os valores basais foram anotados imediatamente antes da injeção de $T Z$, repetindo-se os registros a cada 15 minutos durante 120 minutos. Registraram-se: freqüência cardíaca (FC), pressão arterial média (PAM), débito cardíaco (DC). A partir desses valores, foram calculados índice cardíaco (IC) e sistólico (IS), volume sistólico (VS), trabalho ventricular esquerdo (TVE), resistência periférica total (RPT) e os respectivos índices (ITVE) e (IRPT). Os parâmetros hemodinâmicos mantiveram-se estáveis durante todo o período. Não se observaram variações significativas nos grupos e entre eles. Conclui-se que a TZ estabilizou a função hemodinâmica dos cães com hipovolemia.
\end{abstract}

Palavras-chave: tiletamina-zolazepam, hipovolemia, cães, anestesia dissociativa, hemodinâmica.

\begin{abstract}
Cardiovascular alterations were studied in fourteen mongrel dogs ( $\mathrm{G} 1 \mathrm{n=7}$ control; $\mathrm{G} 2 \mathrm{n=7}$ hypovolemic dogs) under sevoflurane anesthesia (0.5 MAC). Thirty minutes after the induction, blood was removed from right femural artery, equivalent to $3 \%$ of body weight. Thirty minutes past bleeding all hemodynamic parameters were registrated and also were calculated: heart rate (HR), mean arterial pressure (MAP), cardiac output (CO), cardiac index (CI), stroke volume (SV), stroke index (SI), left ventricular stroke (LVS), left ventricular stroke index (LVSI), total peripheric resistance (TPR) and total peripheric resistance index (TPRI) ) and was apllied tiletamine zolazepan (TZ) $10 \mathrm{mg} / \mathrm{kg}$ intravenously. The dogs were monitored during $120 \mathrm{~min}$. In the animals of the group A (no bleeding), was applied the same treatment. Tiletamine zolazepan maintained stable the hemodynamic parameters. It was concluded that tiletamine-zolazepam could be used with security in the anesthesia of the hypovolemic dogs.
\end{abstract}

Keywords: tiletamine-zolazepan, hypovolemia, dogs, dissociative anesthetics, hemodynamic.

\section{Introdução}

As associações anestésicas objetivam diminuir os riscos e os efeitos colaterais inerentes à ação isolada de algumas substâncias anestésicas. A cetamina e a tiletamina promovem a interrupção do fluxo de informações para o córtex sensitivo, deprimindo seletivamente alguns centros cerebrais (Thurmon et al., 1996), caracterizada pela analgesia, manutenção dos reflexos oculares (palpebral e corneano), faríngeo e laríngeo, associado ao aumento do tônus muscular (catalepsia), nistagmo, sialorréia e lacrimejamento (Muir III e Hubbell, 1995).

A ação simpatomimética das ciclohexaminas eleva a freqüência cardíaca e produz vasoconstrição periférica, aumentando a pressão arterial (Paddleford, 1988; Massone, 1994; Muir III e Hubbell, 1995).
Segundo Booth (1992) e Waterman e Lucke (1992), a tiletamina induz efeitos farmacológicos mais potentes e duradouros do que aqueles observados com o uso de outros agentes dissociativos. Chen et al. (1969) notaram que tanto a freqüência cardíaca como a pressão arterial elevaram-se em cães tratados com a tiletamina. Muir III (1993) descreve que, em função das iaracterísticas supracitadas, tal fármaco deverá ser utilizado com cautela em pacientes cardiopatas.

Já o cloridrato de zolazepam possui propriedades sedativa, hipnótica, relaxante muscular e anticonvulsivante, com mínimos efeitos sobre o sistema cardiovascular e respiratório (Hatch et al., 1988; Hellyer et al., 1991; Lin et al., 1993; Muir III e Hubbell, 1995). Alguns benzodiazepínicos podem produzir diminuição da pressão arterial e subseqüente aumento da freqüência cardíaca, sem alterar o débito cardíaco (Rall, 1991).

\footnotetext{
* Aluno do Curso de Doutorado, FCAV/UNESP/Jaboticabal.

** Professor Assistente da Universidade de Marília, SP.

${ }^{\star \star \star}$ Professor Adjunto da Disciplina de Anestesiòlogia Veterinária. Depto. de Clínica e Cirurgia Veterinária. e-mail: valadão@fcav.unesp.br
} 
Os efeitos cardiovasculares da tiletamina/zolazepam variam consideravelmente entre as espécies. Em cães e gatos, a associação produz uma estimulação cardiovascular global, enquanto em macacos, geralmente ocorre depressão. Em cães que receberam TZ em doses de 2-19,8 mg/kg, sem medicação pré-anestésica, foi observado aumento da freqüência cardíaca, visto que, nesta espécie, o débito cardíaco pode aumentar ou manter-se inalterado e quando há aumento, este é atribuído à elevação da freqüência cardíaca (Lin et al., 1993). Descreve-se ainda a resposta bifásica da presssão arterial e resistência vascular periférica, após a injeção intravenosa da TZ. Para Short (1987) e Tracy et al. (1988) a taquicardia promovida pela TZ proporciona uma diminuição do volume sistólico, acompanhado por uma pequena alteração no débito cardíaco, sugerindo que a redução deste parâmetro ocorre, principalmente, como um resultado da diminuição do tempo de enchimento cardíaco.

Estudando a hipovolemia experimental em cães anestesiados com 30mg/kg de cetamina, Nam (1977) registrou o aumento da duração da anestesia, elevação da freqüência cardíaca. Reis-Oliveira et al. (1980) e Haskins e Patz (1990) observaram, após hemorragia experimental, aumento da freqüência cardíaca com diminuição da pressão arterial e do débito cardíaco. A aplicação de cetamina restabeleceu os valores normais do débito cardíaco, da pressão arterial e da freqüência cardíaca.

Apesar dos inúmeros trabalhos já realizados com a associação TZ, a literatura carece de informações consistentes relativas aos efeitos cardiovasculares desta associação, em animais com perda sangüínea por traumas ou por outras causas que induzam hipovolemia.

Desta forma, objetivou-se avaliar os efeitos da tiletamina/ zolazepam sobre os parâmetros hemodinâmicos de cães submetidos à hipovolemia experimental.

\section{Material e método}

Foram utilizados 14 cães, machos, evitando-se fêmeas em gestação ou em estro, adultos, de idade inferior a cinco anos, sem raça definida, vermifugados e mantidos em canis por um período de aproximadamente dois meses, visando estabelecer uma boa condição clínica e nutricional. No período de uma semana antes do início do experimento, os animais foram manipulados e condicionados ao ambiente experimental. Na véspera da realização do experimento, os animais selecionados foram submetidos a um exame clínico e submetidos a um jejum alimentar e hídrico por seis horas.

Para a execução da pesquisa, os cães foram subdivididos aleatoriamente em dois grupos de igual número $(n=7)$, sendo o grupo A constituído por cães normais pesando 11,04 \pm $2,47 \mathrm{~kg}$ e o grupo B com cães submetidos a hipovolemia, pesando $14,26 \pm 2,58 \mathrm{~kg}$.

Cada animal foi mantido sob contenção manual sobre uma mesa cirúrgica e então administrou-se sevofluorano, ${ }^{1}$ por máscara, para a indução anestésica. Após a perda dos reflexos posturais, faríngeos e laríngeos, os animais foram então posicionados em decúbito dorsal e intubados, mantendo-se a anestesia com 1,5 concentração alveolar mínima (CAM) de

\footnotetext{
Sevorane - Lab. Abbot - SP
}

sevofluorano, estabelecida através da leitura da concentração da mistura anestésica expirada, exibida pelo monitor. ${ }^{2}$ Subseqüentemente, após preparo do campo operatório, dissecou-se e cateterizou-se a artéria femoral direita, introduzindo-se um cateter ${ }^{3}$ no seu lúmen, para posteriormente realizar-se a mensuração da pressão arterial. ${ }^{4} \mathrm{~A}$ seguir, após tricotomia e antissepsia da região cervical ventral, procedeuse à dissecação da veia jugular direita, praticando-se uma pequena incisão, que foi suficiente para a introdução do cateter de Swan Ganz, ${ }^{5}$ que foi passado pelo átrio e ventrículo direitos, e posicionado na artéria pulmonar, o qual foi confirmado pela observação do traçado e valores de pressão sangüínea no monitor Dixtal. ${ }^{6}$ Mensurou-se o débito cardíaco por termodiluição, através de injeção de $3 \mathrm{ml}$ de solução sali$\mathrm{na}^{7}$ a $3 \pm 1^{\circ} \mathrm{C}$. O cateter de Swan Ganz foi inflado, quando notava-se no monitor que havia pequenas curvas com picos positivos de grande intensidade, característicos da introdução deste cateter no ventrículo direito (pressão sistólica de 20-30 $\mathrm{mmHg}$ ). Os animais encontravam-se em respiração espontânea durante o procedimento de instrumentalização e inclusive durante as mensurações. Tal procedimento foi repetido por mais duas vezes consecutivas, para cada momento de mensuração do débito cardíaco e tomando-se o cuidado para realizá-lo ao final da expiração. Após ter-se obtido os três valores, determinou-se o valor médio, o qual foi utilizado como valor do débito cardíaco. Em seguida, a concentração alveolar mínima de sevofluorano foi reduzida para 0,5 CAM, aguardando-se um período de 30 minutos para a estabilização da anestesia. Imediatamente após este tempo, procedeu-se à mensuração dos parâmetros cardiorrespiratórios (T-30). Os valores de freqüência cardíaca (FC) (bat/min) foram obtidos através da leitura direta no monitor de eletrocardiografia. ${ }^{8}$ Para a medida da pressão arterial sistólica (PAS), diastólica (PAD) e média (PAM) utilizou-se o método invasivo, através do catéter colocado na artéria femoral, o qual foi conectado ao transdutor do aparelho. Imediatamente antes da injeção de $10 \mathrm{mg} / \mathrm{kg}$, por via intravenosa, de $\mathrm{TZ},{ }^{9}$ foram repetidas as mensurações (T0).

Decorridos 15 minutos da injeção da associação TZ (T15), os mesmos parâmetros foram reavaliados e, subseqüentemente, repetiu-se tais aferições com intervalos de 15 minutos, até o tempo de 120 minutos (T120). Durante o procedimento experimental os animais receberam solução de ringer lactato ${ }^{10}$ por via intravenosa, na dose de $5 \mathrm{ml} / \mathrm{kg} / \mathrm{hora}$.

Os animais do grupo $B$ foram manipulados experimentalmente de forma idêntica aos do grupo A porém, decorrido o tempo de estabilização da anestesia com sevofluorano em 0,5 CAM, foi procedida a hipovolemia, retirando-se um volume de sangue arterial equivalente à $3 \%$ de peso corpóreo, através do catéter aplicado na artéria femoral. Imediatamente após a retirada do sangue, procedeu-se à leitura dos parâmetros (TO) e aplicou-se a associação TZ na mesma dose e via utili-

\footnotetext{
${ }^{2}$ POET IQ - Criticare - WI, USA

316 G-Abocart - Lab. Abbot - SP

${ }^{4}$ Digimax - Digicare - RJ

5 Swan Ganz 5 F - Baxter - USA

- Dixtal Mod. 2010 com módulo para débito cardiaco

7 Soro fisiológico - Aster - Sorocaba, SP

${ }^{8}$. Dixtal Mod. 2010 com módulo para eletrocardiografia

9 Zoletil - Virbac - SP

${ }^{10}$ Soro ringer com lactato - Aster - Sorocaba, SP
} 
zada para o grupo A. A partir deste momento, repetiram-se as leituras dos parâmetros nos mesmos intervalos já descritos para o grupo $A$.

Procedeu-se à análise de variância e os valores médios de cada tempo no mesmo grupo foram comparados entre si, aplicando-se o teste de Student-Newman-Keuls com nível de significância $p<0,05$. Para a comparação dos valores médios de cada momento entre os grupos foi utilizado o teste t de Student.

\section{Resultados}

No grupo $B$ a freqüência cardíaca permaneceu estável até 0 final do período de observação. No grupo A, apesar da freqüência cardíaca ter apresentado valores nominalmente maiores após a aplicação da TZ, também não foi registrado aumento significativo deste parâmetro.

A pressão arterial média não se alterou significativamente nos dois grupos, embora no grupo B tenha sido restabelecido o mesmo valor basal após a injeção da TZ. Nas comparações entre os grupos não foram observadas diferenças entre os valores médios anotados para a pressão arterial média.

No grupo A o débito cardíaco manteve-se acima dos valores iniciais, sendo $33 \%$ maior durante os primeiros 30 minutos. No grupo $B$, após a retirada de sangue, o débito cardíaco foi $50 \%$ menor, apesar desta redução não ter sido estatisticamente significativa. Neste grupo a aplicação da TZ aumentou o débito cardíaco em $53 \%$, enquanto no A o DC aumentou em $31 \%$. Não foram observadas alterações significati- vas neste parâmetro, nas comparações dentro dos grupos ou entre eles.

$O$ índice cardíaco manteve-se acima dos valores iniciais, sendo $33 \%$ maior durante os primeiros 30 minutos no grupo $A$. Já no grupo $B$, houve um aumento de $30 \%$ em relação ao T0. Foi constatada uma redução do IC, no grupo hipovolêmico, em relação ao normal nos intervalos T0, T30, T45, T60.

O volume sistólico diminuiu gradativamente no grupo normovolêmico com o decorrer do tempo, apresentando um padrão inverso ao observado no grupo hipovolêmico, porém não foram constatados significância estatística nos grupos ou entre eles.

O índice sistólico nos cães hipovolêmicos foi significativamente menor em T0 comparado ao T120. Também foi menor em relação ao TO do grupo normal.

Não existiram alterações significativas entre os momentos ou grupos para a RPT e o IRPT. Observou-se no grupo B um aumento nominal dos valores para RPT durante os primeiros 15 minutos, já no grupo A constatou-se uma resposta bifásica com queda inicial, e tendência a uma aproximação dos valores basais.

O trabalho ventricular esquerdo e o seu índice permaneceram iguais entre os grupos, porém notou-se uma predisposição para elevação durante os primeiros 15 minutos do grupo hipovolêmico, com valores acima do T0 no decorrer do tempo e superiores em relação ao grupo normovolêmico.

Tabela 1: Valores médios e desvio-padrão de freqüência cardíaca (FC), pressão arterial média (PAM), débito cardíaco (DC), índice cardiaco (IC), volume sistólico (VS), índice sistólico (IS), resistência periférica total (RPT), índice de resistência periférica total (IRPT), trabalho ventricular esquerdo (TVE) e índice de trabalho ventricular esquerdo (ITVE) em cães normovolêmicos (N) ou com hipovolemia $(H)$, tratados com a associação tiletamina-zolazepam (10 mg/kg) pela via intravenosa, Jaboticabal (1999)

\begin{tabular}{|c|c|c|c|c|c|c|c|c|c|c|c|c|c|c|c|c|c|c|c|c|}
\hline \multirow[t]{2}{*}{$\begin{array}{c}\text { Tempo } \\
(\mathrm{min})\end{array}$} & \multicolumn{2}{|c|}{$\begin{array}{c}\text { FC } \\
\text { (batim. } / \mathrm{min})\end{array}$} & \multicolumn{2}{|c|}{$\begin{array}{c}\text { PAM } \\
(\mathrm{mmHg})\end{array}$} & \multicolumn{2}{|c|}{$\begin{array}{c}\text { DC } \\
\text { (litros } / \mathrm{min})\end{array}$} & \multicolumn{2}{|c|}{$\begin{array}{c}\text { IC } \\
\left.\text { (litros } / \mathrm{min}^{2} \mathrm{~m}^{2}\right)\end{array}$} & \multicolumn{2}{|c|}{$\begin{array}{c}\text { VS } \\
\text { (ml/batimento) }\end{array}$} & \multicolumn{2}{|c|}{$\begin{array}{c}\text { IS } \\
\left(\mathrm{ml} / \mathrm{batim} / \mathrm{m}^{2}\right)\end{array}$} & \multicolumn{2}{|c|}{$\begin{array}{c}\text { RPT } \\
\left(\text { dinaxseg/cm }{ }^{5}\right)\end{array}$} & \multicolumn{2}{|c|}{$\begin{array}{c}\text { IRPT } \\
\left(\text { dinaxseg } / \mathrm{cm}^{5} \mathrm{xm}^{2}\right)\end{array}$} & \multicolumn{2}{|c|}{$\begin{array}{c}\text { TVE } \\
(\mathrm{kg} \times \mathrm{m} / \mathrm{min} .)\end{array}$} & \multicolumn{2}{|c|}{$\begin{array}{c}\text { ITVE } \\
\left(\mathrm{kg} \times \mathrm{m} / \mathrm{min} \times \mathrm{m}^{2}\right)\end{array}$} \\
\hline & $\mathrm{N}$ & $H$ & $\mathrm{~N}$ & H & $\mathbf{N}$ & H & $\mathbf{N}$ & $\mathrm{H}$ & $\mathrm{N}$ & $H$ & $\mathrm{~N}$ & $\mathrm{H}$ & $\mathrm{N}$ & $\mathrm{H}$ & $N$ & $\mathrm{H}$ & $\mathbf{N}$ & H & $N$ & $H$ \\
\hline-30 & $\begin{array}{l}104 \\
{[36]}\end{array}$ & $\begin{array}{l}128 \\
{[29\}}\end{array}$ & $\begin{array}{c}67 \\
{[16]}\end{array}$ & $\begin{array}{l}83 \\
{[20]}\end{array}$ & $\begin{array}{c}1,67 \\
{[0,52]}\end{array}$ & $\begin{array}{c}2.03 \\
{[0.57]}\end{array}$ & $\begin{array}{c}3.40 \\
{[0,89]}\end{array}$ & $\begin{array}{c}3.51 \\
{[0,81]}\end{array}$ & $\begin{array}{l}17,52 \\
{[6,35]}\end{array}$ & $\begin{array}{l}16,31 \\
{[3,92]}\end{array}$ & $\begin{array}{c}37,10 \\
{[15,29]}\end{array}$ & $\begin{array}{l}28,21 \\
{[5,83]}\end{array}$ & $\begin{array}{c}3612,01 \\
{[1629,60]}\end{array}$ & $\begin{array}{l}3426,24 \\
{[811,99]}\end{array}$ & $\begin{array}{c}7782,95 \\
{[4072,62]}\end{array}$ & $\begin{array}{c}6164,63 \\
{[1996,14]}\end{array}$ & $\begin{array}{c}1,50 \\
{[0,55]}\end{array}$ & $\begin{array}{c}2,37 \\
{[0,95]}\end{array}$ & $\begin{array}{c}3,09 \\
{[1,20]}\end{array}$ & $\begin{array}{c}4,08 \\
{[1,53]}\end{array}$ \\
\hline 0 & $\begin{array}{l}104 \\
{[36]}\end{array}$ & $\begin{array}{l}116 \\
{[27]}\end{array}$ & $\begin{array}{c}67 \\
{[16]} \\
\end{array}$ & $\begin{array}{l}66 \\
{[24]}\end{array}$ & $\begin{array}{c}1,67 \\
{[0,52]}\end{array}$ & $\begin{array}{c}1,16 \\
{[0,38]}\end{array}$ & $\begin{array}{c}3,40 \\
{[0,89]}\end{array}$ & $\begin{array}{l}2,29^{*} \\
{[0,78]}\end{array}$ & $\begin{array}{l}17,52 \\
{[6,35]}\end{array}$ & $\begin{array}{l}12,79 \\
{[3,66]}\end{array}$ & $\left.\begin{array}{c}37,10 \\
{[15,29]}\end{array}\right]$ & $\begin{array}{c}20,08 \# \\
{[6,29]} \\
\end{array}$ & $\begin{array}{c}3612,01 \\
{[1629,60]}\end{array}$ & $\begin{array}{c}4193,97 \\
{[1643,17]}\end{array}$ & $\begin{array}{c}7782,95 \\
{[4072,62]}\end{array}$ & $\begin{array}{c}7509,24 \\
{[3430,89]}\end{array}$ & $\begin{array}{c}1,50 \\
{[0,55]}\end{array}$ & $\begin{array}{c}1,20 \\
{[0,54]}\end{array}$ & $\begin{array}{c}3.09 \\
{[1,20]}\end{array}$ & $\begin{array}{c}2.11 \\
{[1,05]} \\
\end{array}$ \\
\hline 15 & $\begin{array}{l}134 \\
{[26]}\end{array}$ & $\begin{array}{l}120 \\
{[27]}\end{array}$ & $\begin{array}{c}70 \\
{[12]}\end{array}$ & $\begin{array}{l}86 \\
{[26]}\end{array}$ & $\begin{array}{c}2,19 \\
{[0,77]}\end{array}$ & $\begin{array}{c}1,78 \\
{[0,68]}\end{array}$ & $\begin{array}{c}4,46 \\
{[1,41]}\end{array}$ & $\begin{array}{c}3,03 \\
{[1,01]}\end{array}$ & $\begin{array}{l}16,56 \\
{[5,11]}\end{array}$ & $\begin{array}{l}14,89 \\
{[5,32]}\end{array}$ & $\begin{array}{l}33,87 \\
{[9,54]}\end{array}$ & $\begin{array}{l}25,71 \\
{[8,86]}\end{array}$ & $\begin{array}{c}2733,71 \\
{[1344,52]}\end{array}$ & $\begin{array}{c}4312,03 \\
{[1643,17]}\end{array}$ & $\begin{array}{c}5841,17 \\
{[3289,41]}\end{array}$ & $\begin{array}{c}7271,71 \\
{[4002,54]}\end{array}$ & $\begin{array}{c}2,09 \\
{[0,93]}\end{array}$ & $\begin{array}{c}2,15 \\
{[1,08]}\end{array}$ & $\begin{array}{c}4,27 \\
{[1,76]}\end{array}$ & $\begin{array}{c}3,68 \\
{[1,92]}\end{array}$ \\
\hline 30 & $\begin{array}{l}136 \\
{[19]}\end{array}$ & $\begin{array}{l}116 \\
{[15]}\end{array}$ & $\begin{array}{c}69 \\
{[14]}\end{array}$ & $\begin{array}{c}74 \\
{[20]}\end{array}$ & $\begin{array}{c}2,23 \\
{[0,72]}\end{array}$ & $\begin{array}{c}1,67 \\
{[0,53]}\end{array}$ & $\begin{array}{c}4,59 \\
{[1,49]}\end{array}$ & $\begin{array}{l}2,87^{*} \\
{[0,78]}\end{array}$ & $\begin{array}{l}16,90 \\
{[5,98]}\end{array}$ & $\begin{array}{l}14,34 \\
{[4,30]}\end{array}$ & $\begin{array}{c}34,85 \\
{[12,83]}\end{array}$ & $\begin{array}{l}24,85 \\
{[6,89]}\end{array}$ & $\begin{array}{c}2908,82 \\
{[1806,90]}\end{array}$ & $\begin{array}{c}3814,34 \\
{[1381,57]}\end{array}$ & $\begin{array}{c}6267,77 \\
{[4382,45]}\end{array}$ & $\begin{array}{c}6546,72 \\
{[3398,01]}\end{array}$ & $\begin{array}{c}2,04 \\
{[0,84]}\end{array}$ & $\begin{array}{c}1,67 \\
{[0,69]}\end{array}$ & $\begin{array}{c}4,25 \\
{[1,89]}\end{array}$ & $\begin{array}{c}2,89 \\
{[1,19]}\end{array}$ \\
\hline 45 & $\begin{array}{c}129 \\
{[13]}\end{array}$ & $\begin{array}{l}112 \\
{[20]}\end{array}$ & $\begin{array}{l}64 \\
{[14]}\end{array}$ & $\begin{array}{c}70 \\
{[18]}\end{array}$ & $\begin{array}{c}2,04 \\
{[0,56]}\end{array}$ & $\begin{array}{c}1,56 \\
{[0,43]}\end{array}$ & $\begin{array}{c}4,21 \\
{[1,17]}\end{array}$ & $\begin{array}{c}2,69^{*} \\
{[0,61]}\end{array}$ & $\begin{array}{l}15,76 \\
{[3,73]}\end{array}$ & $\begin{array}{l}14,18 \\
{[4,24]}\end{array}$ & $\begin{array}{l}32,61 \\
{[8,06]}\end{array}$ & $\begin{array}{l}24,64 \\
{[7,54]}\end{array}$ & $\begin{array}{l}2649,63 \\
{[813.04]}\end{array}$ & $\begin{array}{c}3836,49 \\
{[1384,04]}\end{array}$ & $\begin{array}{c}5608,35 \\
{[2191,66]}\end{array}$ & $\begin{array}{c}6575,58 \\
{[3467,87]}\end{array}$ & $\begin{array}{c}2,04 \\
{[0,93]}\end{array}$ & $\begin{array}{c}1,47 \\
{[0,47]}\end{array}$ & $\begin{array}{c}4,29 \\
{[2,18]}\end{array}$ & $\begin{array}{r}2,55 \\
{[0.85]}\end{array}$ \\
\hline 60 & $\begin{array}{l}122 \\
{[14]}\end{array}$ & $\begin{array}{l}108 \\
{[19]}\end{array}$ & $\begin{array}{l}65 \\
{[14]}\end{array}$ & $\begin{array}{c}68 \\
{[13]}\end{array}$ & $\begin{array}{c}1,95 \\
{[0,58]}\end{array}$ & $\begin{array}{c}1,56 \\
{[0,43]}\end{array}$ & $\begin{array}{c}3,99 \\
{[1,04]}\end{array}$ & $\begin{array}{l}2,70^{*} \\
{[0,64]}\end{array}$ & $\begin{array}{l}15,80 \\
{[3,36]}\end{array}$ & $\begin{array}{l}14,74 \\
{[4,30]}\end{array}$ & $\begin{array}{l}32,50 \\
{[5,61]}\end{array}$ & $\begin{array}{l}25,73 \\
{[7,95]}\end{array}$ & $\begin{array}{l}2747,91 \\
{[552,65]}\end{array}$ & $\begin{array}{c}3740,88 \\
{[1337,41]}\end{array}$ & $\begin{array}{c}5579,50 \\
{[1790,36]}\end{array}$ & $\begin{array}{c}6371,47 \\
{[3234,10]} \\
\end{array}$ & $\begin{array}{c}1,85 \\
{[0,95]} \\
\end{array}$ & $\begin{array}{c}1,42 \\
{[0,40]}\end{array}$ & $\begin{array}{c}3,62 \\
{[1,75]}\end{array}$ & $\begin{array}{c}2,46 \\
{[0,64]}\end{array}$ \\
\hline 75 & $\begin{array}{l}121 \\
{[13]}\end{array}$ & $\begin{array}{l}109 \\
{[18]}\end{array}$ & $\begin{array}{l}67 \\
{[14]}\end{array}$ & $\begin{array}{c}73 \\
{[18]}\end{array}$ & $\begin{array}{c}1,79 \\
10.561\end{array}$ & $\begin{array}{c}1,65 \\
10.39]\end{array}$ & $\begin{array}{c}3,66 \\
{[0,97]}\end{array}$ & $\begin{array}{c}2,86 \\
{[0,58]}\end{array}$ & $\begin{array}{l}14,54 \\
{[3,24]}\end{array}$ & $\begin{array}{l}15,43 \\
{[3,98]}\end{array}$ & $\begin{array}{l}29,89 \\
{[5,58]}\end{array}$ & $\begin{array}{l}27,01 \\
{[7,56]}\end{array}$ & $\begin{array}{l}3094,08 \\
{[632,06]}\end{array}$ & $\begin{array}{c}3679,38 \\
|1164,73|\end{array}$ & $\begin{array}{c}6311,06 \\
{[2141,63]}\end{array}$ & $\begin{array}{c}6165,32 \\
\mid 2753,431 \\
\end{array}$ & $\begin{array}{c}1,68 \\
{[0,82]}\end{array}$ & $\begin{array}{c}1,61 \\
{[0,53]}\end{array}$ & $\begin{array}{c}3,39 \\
{[1,53]}\end{array}$ & $\begin{array}{r}2,79 \\
{[0,83]}\end{array}$ \\
\hline 90 & $\begin{array}{l}120 \\
{[17]}\end{array}$ & $\begin{array}{l}109 \\
{[18]}\end{array}$ & $\begin{array}{l}66 \\
{[17]}\end{array}$ & $\begin{array}{c}72 \\
{[13]}\end{array}$ & $\begin{array}{r}1,66 \\
{[0,62]}\end{array}$ & $\begin{array}{c}1,77 \\
{[0,58]}\end{array}$ & $\begin{array}{c}3,35 \\
{[0,97]}\end{array}$ & $\begin{array}{c}3,07 \\
{[0,89]}\end{array}$ & $\begin{array}{l}14,15 \\
{[4,39]}\end{array}$ & $\begin{array}{l}16,45 \\
{[5,93]}\end{array}$ & $\begin{array}{l}29,03 \\
{[8,21]}\end{array}$ & $\begin{array}{l}28,71 \\
{[9,62]} \\
\end{array}$ & $\begin{array}{l}3404,73 \\
{[934,96]} \\
\end{array}$ & $\begin{array}{c}3560,37 \\
{[1316,51]} \\
\end{array}$ & $\begin{array}{c}6989,49 \\
{[2815,34]}\end{array}$ & $\begin{array}{c}5690,33 \\
{[2752,86]} \\
\end{array}$ & $\begin{array}{r}1,58 \\
{[0.98]} \\
\end{array}$ & $\begin{array}{r}1,69 \\
{[0,47]} \\
\end{array}$ & $\begin{array}{r}3,16 \\
{[1,73]} \\
\end{array}$ & $\begin{array}{r}2,92 \\
{[0,71]} \\
\end{array}$ \\
\hline 105 & $\begin{array}{c}118 \\
{[17]}\end{array}$ & $\begin{array}{l}107 \\
{[13]}\end{array}$ & $\begin{array}{l}66 \\
{[14]}\end{array}$ & $\begin{array}{c}69 \\
{[11]}\end{array}$ & $\begin{array}{c}1,73 \\
{[0,71]}\end{array}$ & $\begin{array}{c}1,70 \\
{[0,59]}\end{array}$ & $\begin{array}{c}3,47 \\
{[1,04]}\end{array}$ & $\begin{array}{c}2,94 \\
{[0,92]}\end{array}$ & $\begin{array}{l}14,27 \\
{[4,08]}\end{array}$ & $\begin{array}{l}16,45 \\
{[5,23]}\end{array}$ & $\begin{array}{l}29,02 \\
{[5,40]}\end{array}$ & $\begin{array}{l}27,56 \\
{[8,50]}\end{array}$ & $\begin{array}{c}3337,39 \\
{[1134,00]}\end{array}$ & $\begin{array}{c}3601,79 \\
{[1405,81]}\end{array}$ & $\begin{array}{c}6949,65 \\
{[3323,90]}\end{array}$ & $\begin{array}{c}6121,25 \\
{[2753,43]}\end{array}$ & $\begin{array}{c}1.69 \\
{[0,95]} \\
\end{array}$ & $\begin{array}{c}1,54 \\
{[0,46]}\end{array}$ & $\begin{array}{c}3,39 \\
{[1,49]}\end{array}$ & $\begin{array}{c}2,66 \\
{[0,68]}\end{array}$ \\
\hline 120 & $\begin{array}{c}117 \\
{[18]}\end{array}$ & $\begin{array}{l}104 \\
{[12]}\end{array}$ & $\begin{array}{l}68 \\
{[14]}\end{array}$ & $\begin{array}{c}63 \\
{[12]}\end{array}$ & $\begin{array}{c}1,71 \\
{[0,67]}\end{array}$ & $\begin{array}{c}1,78 \\
{[0,56]}\end{array}$ & $\begin{array}{c}3,40 \\
{[1,01]}\end{array}$ & $\begin{array}{c}3,09 \\
{[0,92]}\end{array}$ & $\begin{array}{l}14,26 \\
{[3,72]}\end{array}$ & $\begin{array}{l}15,84 \\
{[4,92]}\end{array}$ & $\begin{array}{l}29,14 \\
{[5,64]}\end{array}$ & $\begin{array}{c}30,20 \# \\
{[9,57]}\end{array}$ & $\begin{array}{c}3489,65 \\
{[1206,47]}\end{array}$ & $\begin{array}{c}3094,92 \\
{[1078,16]}\end{array}$ & $\begin{array}{c}7261,68 \\
{[3512,74]}\end{array}$ & $\begin{array}{c}5168,02 \\
{[2154,92]}\end{array}$ & $\begin{array}{c}1,70 \\
{[0,88]}\end{array}$ & $\begin{array}{c}1,49 \\
{[0,42]}\end{array}$ & $\begin{array}{c}3.43 \\
{[1,41]}\end{array}$ & $\begin{array}{c}2,57 \\
{[0,64]}\end{array}$ \\
\hline
\end{tabular}

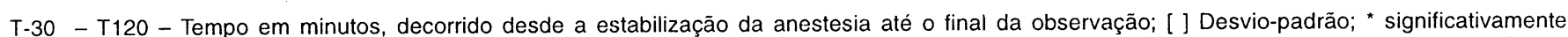

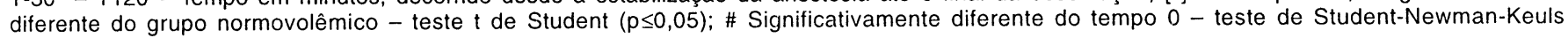
$(p \leq 0,05)$ 


\section{Discussão}

Não é incomum que a perda sangüínea ou a inadequada perfusão tecidual, que caracterizam o choque, ocorram em cães (Waddell et al., 1998). A indução anestésica intravenosa em pacientes cirúrgicos nestas condições representa um desafio, principalmente pelo risco de depressão cardiovascular. As alterações hemodinâmicas, por ação dos anestésicos, sujeita os pacientes cirúrgicos emergenciais ao agravamento do quadro de hipovolemia, quase sempre por induzirem hipotensão (Nam, 1977; Reis-Oliveira et al., 1980; Ingwersen et al., 1988; Haskins e Patz, 1990).

A cetamina aumenta a freqüência cardíaca e a pressão arterial por estimular uma atividade simpatomimética (Short, 1987; Hellyer et al., 1991; Massone, 1994; Paddleford, 1999). A tiletamina, quando injetada isoladamente por via intravenosa, também produz aumento da freqüência cardíaca $e$ da pressão arterial (Soma, 1971). Em associação com o zolazepam, este agente dissociativo, segundo Tracy et al. (1988) e Almeida (1998), induz taquicardia e diminuição da pressäo arterial, podendo ou não alterar o débito cardíaco, relacionado ao aumento do tônus simpático por estimulação do sistema nervoso central ou, talvez, por um decréscimo do tônus vagal (Mcgrath et al., 1975). Tem sido relatado que a tiletamina é a responsável pelo efeito bifásico sobre a pressão arterial após a injeção intravenosa (Hellyer et al., 1989; Lin et al., 1993). Controversamente, neste estudo, após a injeção da TZ em cães normovolêmicos, não foram observadas alterações na pressão arterial média. Destaca-se que a associação de cetaminadiazepam, duas substâncias de grupos farmacológicos semelhantes ao do nosso estudo, aumentou a pressão arterial de cães (Newell, 1997). Considerando a estabilidade circulatória produzida pela TZ Potoczac e Corey (1975) e Hess (1991) descreveram que estes fármacos não interferem de forma significativa com a pressão arterial. Assim, nos cães hipovolêmicos tratados com a associação, os valores relativos a pressão arterial média mostraram-se semelhantes àqueles observados para os cães normais. Este achado pode ser considerado como favorável, desde que a associação não potencializou o quadro de hipovolemia.

Os valores da freqüência cardíaca foram superiores aos registrados inicialmente, muito embora não existam relatos sobre os efeitos da TZ em cães hipovolêmicos, apesar da consonância dos relatos de Fieni et al. (1988), Genevois et al. (1988), que descreveram o aumento da freqüência cardiaca depois da injeção da TZ em cães com volemia normal. Por sua vez, Haskins e Patz (1990) descreveram ser a cetamina uma alternativa para a indução de anestesia em pacientes potencialmente hipovolêmicos, por manter a função cardiovascular.

O débito cardíaco é determinado pela freqüência cardiaca e pelo volume sistólico, podendo ser alterado por fatores que modulem o cronotropismo ou volume de ejeção (Haskins, 1987; Ganong, 1991; Sisson, 1992, Thibodeau e Patton, 1993). As alterações decorrentes no DC podem ser explicadas pelo fato de que a associação TZ pode aumentar ou manter inalterado este parâmetro e, quando há aumento, este é atribuído à elevação da FC (Short, 1987; Lin et al., 1993). De fato, a aplicação de TZ nos cães normovolêmicos não reduziu o DC e nos cães hipovolêmicos o débito diminuiu após a hemorragia, mas restabeleceu-se dentro de 15 minutos da aplicação do fármaco. Tal situação, muito provavelmente tenha ocorrido em função da manutenção da FC. Por outro lado, a diminuição do índice cardíaco, no grupo hipovolêmico, pode ser explicada pela padronização desse índice em relação à área de superfície corporal dos animais.

A redução no volume sistólico do grupo normovolêmico poderia ser atribuída diretamente ao aumento da FC induzida pela $T Z$, que embora não tenha alterado o $D C$, pode reduzir o VS por diminuição do tempo de preenchimento cardíaco (Short, 1987; Tracy et al., 1988). Por outro lado, nos animais hipovolêmicos o VS aumentou em razão direta da diminuição da FC.

A redução nos valores de IRPT e RPT, no grupo normovolêmico poderia ser atribuída a um aumento nominal dos valores de DC com a manutenção de uma estabilidade da PAM. Nota-se uma elevação da RPT durante os primeiros 15 minutos nos cães hipovolêmicos provavelmente incitada pela resposta simpato-adrenal, confluente com os mecanismos compensatórios de homeostase (Reis-Oliveira et al., 1980; Ingwersen et al., 1988; Haskins e Patz, 1990).

Apesar da inexistência estatística, os valores de TVE e o seu índice foram nominalmente menores para o grupo hipovolêmico, muito provavelmente em razão direta dos valores superiores de débito cardíaco em favor dos cães normovolêmicos, bem como a proximidade dos valores de pressão arterial média ocorrida entre os grupos.

\section{Conclusões}

Após a realização deste estudo, pôde-se concluir que a associação TZ manteve uma estabilidade hemodinâmica, nos cães com hipovolemia.

\section{Agradecimentos}

À Fundação de Amparo e Pesquisa do Estado de São Paulo (FAPESP - Processo n 96/12830-0).

\section{Referências}

ALMEIDA, E.M.P. Efeitos cardiorrespiratórios da associação de tiletamina e zolazepam em cães (Canis familiaris) pré-tratados ou não pela acepromazina.1998. 137 f. Dissertação (Mestrado) - Faculdade de Ciências Agrárias e Veterinárias, Universidade Estadual Paulista, 1998.
BOOTH, N.H. Anestésicos intravenosos e outros colaterais. In: BOOTH, N.H.; McDONALD, L.E. Farmacologia e Terapêutica em Veterinária. 6. ed. Rio de Janeiro: Guanabara Koogan, 1992. p.168-218.

CHEN, G.; ENSOR, C.R.; BOHNER, B. The pharmacology of 2-(etylamino)2-(2-thienyl)-cyclohexanone- $\mathrm{HCl}(\mathrm{Cl} 634)$. J. Pharm. Exp. Thera., v. 168, p. $171-178,1969$. 
FIENI, F.; TAINTURIER, D.; DENISSEL, E.; KLETHI, H. Uso da associação tiletamina-zolazepam pela via endovenosa na anestesia do cão. Hora veterinária, v. 8, n. 45, p. 45-48, 1988.

GANONG, W.F. O coração como uma bomba. In: GANONG, W.F. Fisiologia Médica. 15 ed. Rio de Janeiro: Prentice-Hall doBrasil, 1991. p. 399-407.

GENEVOIS, J.P.; AUTEFAGE, A.; FAYOLLE, A.; CAZIEUX, A.; COMBES, F. Etude comparée des effects des associations xilazyne-ketamine et tiletamine-zolazepam sur quelques grants fonctions chez le chien. Recueil du medicine veterinaire, v. 164, p. 289-296, 1988.

HASKINS, S.C. Monitoring the anesthetized patiente. In: SHORT, C.E. Principle e practice of veterinary anesthesia. Baltimore: Willians e Wilkins, 1987. p. 455-477.

HASKINS, S.C.; PATZ, J.D. Ketamine in hypovolemic dogs. Critical care medicine, v. 18, n. 6, p. 625-629, 1990.

HATCH, R.C.; CLARK, J.D.; JERNIHAN, A.D.; TRACY, C.H. Searching for a save, effective antagonist to Telazol overdose. Veterinary medicine, v. 83, p. 112-117, 1988.

HELLYER, P.W.; MUIR III, W.W.; HUBBELL, J.A.E. Cardiorespiratory effects of the intravenous administration of tiletamine-zolazepam to dogs. Veterinary surgery, v. 18, p. 160-165, 1989.

HELLYER, P.W.; FREEMAN., L.C.; HUBBELL, J.A.E. Induction of anesthesia with diazepan-ketamine and midazolam-ketamine in greyhounds. Veterinary surgery, v. 20, n. 2, p. 143-147, 1991.

HESS, L. Anaesthesia in the dog with Tilest 500 . Tierarztliche praxis, v. 19, n. 1, p. 107-110, 1991.

INGWERSEN, W.; ALLEN, D.G.; DYSON, D.H.; BLACK, W.D.; MARK, T.G.; VALLIANT, A.E. Cardiopulmonary effects of a ketamine/acepromazine combination in hypovolemic cats. Canadian journal of veterinary research, v. 52, n. 4, p. 423-427, 1988.

LIN, H.C.; THURMON, J.C.; BENSON, G.J.; TRANQUILLI, W.J. Telazol-a review of its pharmacology and use in veterinary medicine. Journal of veterinary pharmacology and therapeutic, v. 16, p. 383-418, 1993.

LUMB, W. V.; JONES, E. W. Veterinary anesthesia. Baltimore: Williams \& Wilkins, 1996. 927 p.

McGRATH, J.C.; MacKENZIE, J.E.; MILLER, R.A. Effects of ketamine on central sympathetic discarge and baroreceptor reflex durin mechanical ventilation. British journal of anaesthesia. v. 47, p. 1141-1147, 1975.

MASSONE, F. Anestesiologia Veterinária. Rio de Janeiro: Guanabara Koogan, 1994. 252 p.

MUIR III, W.W. Anesthetic and techniques. In: SLATTER, D. Textbook of small animal surgery. 2. ed. Philadelphia: W.B. Saunders, 1993. p. 2245-2251.

MUIR III, W.W.; HUBBELL, J.A.E. Specific intravenous anesthetic drugs. In: MUIR III, W.W.; HUBBELL, J.A.E. Handbook of Veterinary Anesthesia. 2. ed. St. Louis: Mosby. 1995. p. 74-86.
NAM, T.C. Studies on the effects of ketamine hydrochloride on dogs after bleeding. Japanese journal of veterinary science, v. 39, p. 273281, 1977.

NEWELL, S.M.; KO, J.C.; GINN, P.E.; HEATON, J.T.G.; HYATT, D.A.; CARDWELL, A. L.; MAURAGIS, D.F.; HARRISON, J.M. Effects of three sedative protocols on glomerular filtration rate in clinically normal dogs. American journal of veterinary research, v. 58, n. 5, p. 446450, 1997.

PADDLEFORD, R.R. General anesthesia. In: PADDLEFORD, R.R.Manual of small animal anesthesia. New York: Churchill Livingstone, 1988. p. 31-73.

PADDLEFORD, R.R. Anesthetic agents. In: PADDLEFORD, R.R.Manual of small animal anesthesia. 2. ed. Philadelphia: Saunders, 1999. p. 31-77.

POTOCZAK, R.; COREY, R. The effects of CL-744 upon cardiovascular function in the dog. Federation proceedings, v. 34, p. 771, 1975.

RALL, T.W. Hipnóticos e sedativos. In: GILMAN, A.G.; RALL, T.W.; NIES, A.S. Goodman e Gilman. As bases farmacológicas da terapêutica. 8. ed. Rio de Janeiro: Guanabara Koogan, 1991. p. 228252.

REIS-OLIVEIRA, M.P.; NICOLETTI, R.L:; FELÍCIO, A.A.; FERRAZ, A.L.M. Efeitos cardiovasculares da quetamina em cães hipovolêmicos. $R e-$ vista brasileira de anestesiologia, v. 30, n. 3, p. 167-172, 1980.

SHORT, C.E. Dissociative anesthesia. In: SHORT, C.E. Principles e Practice of Veterinary Anesthesia. Baltimore, Williams e Wilkins, 1987. p. 158-169.

SISSON, D. Avaliação clínica da função cardiaca. In: ETTINGER, S.J. Tratado de medicina interna veterinária. 3. Ed. São Paulo: Manole, 1992. p. 970-986.

SOMA, L.R. Textbook of veterinary anesthesia. Baltimore: Williams e Wilkins, 1971, $621 \mathrm{p}$.

THIBODEAU, G.A.; PATTON, K.T. Physilogy of the cardiovascular system. In: THIBODEAU, G.A.; PATTON, K.T. Anatomy e physiology. 2. ed. St. Louis: Mosby, 1993 , p. 510-529.

TRACY, C.H.; SHORT, C.E.; CLARK, B.C. Comparing the effects of intravenous and intramuscular administration of Telazol. Veterinary medicine, v. 83, p. 104-111, 1988.

THURMON, J.C.; TRANQUILLI, W.J.; BENSON, G.J.(ed.). Lumb \& Jones Veterinary Anesthesia. Baltimore: Williams e Wilkins, 1996. 927 p.

WADDELL, L.S.; DROBATZ, K.J.; OTTO, C.M. Corticosteroids in hypovolemic shock. Compendium on continuing education for the practicing veterinarian. v. 20, n. 5, p. 571-588, 1998.

WATERMAN, A.E.; LUCKE, J.N. Anestesia intravenosa. In: HILBERY, A.D.R. Manual de anestesia de los pequeños animales. 3. ed. Zaragoza: Acribia, 1992. p. 45-56. 